BIOGEOCHEMISTRY

\section{Water from a stone}

Water is necessary for life, yet life persists in even the most extreme arid environments. In the Atacama Desert of Chile, the driest non-polar region on Earth, colonies of endolithic microbes marble the rocks, impervious to the deadly low humidity levels. The only way for these endoliths to survive in the Atacama is to extract water from the rocks in which they live. Their mechanisms of water uptake, however, are poorly understood.

David Kisailus from the University of California Irvine, USA, and colleagues, grew a cyanobacterium isolated from the Atacama Desert on gypsum. Gypsum, a common rock in the Atacama, is composed of hydrated calcium sulfate. Electron microscopy revealed that when incubated under dry conditions (no growth medium or

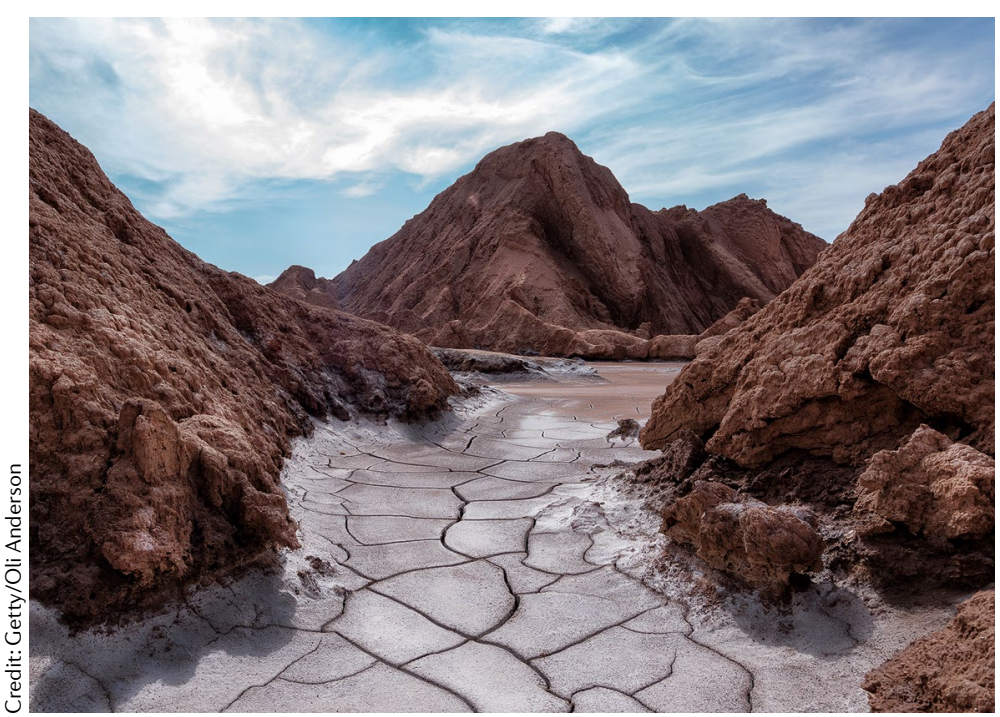

liquid added), the cyanobacterium proliferated and etched away portions of the gypsum. X-ray diffraction showed that the etched gypsum had been dehydrated to anhydrite, indicating that water was liberated through a mineralogical conversion. Cyanobacterial secretion of an acidic extracellular matrix was detected by FTIR spectroscopy and might have altered the chemical structure of the rock resulting in the extraction of water.

The ubiquity of mineralogical water extraction mechanisms across other organisms or rock types in the Atacama remains to be determined as the experiments were limited to just one species and a single mineral. However, a flowering plant found in Spain employs a similar strategy to survive dry summers, so this mechanism could be widespread. The implications of microbes pulling water from rocks extends beyond Earth, highlighting a way that extraterrestrial life might eke out a living in other extreme environments such as those on Mars.

Kyle R. Frischkorn Nature Communications

ORIGINAL ARTICLE Huang, W. et al. Mechanism of water extraction from gypsum rock by desert colonizing microorganisms. Proc. Natl Acad. Sci. USA 117, 10681-10687 (2020) 